\title{
Proportional-Integral Degradation Control Allows Accurate Tracking of Biomolecular Concentrations With Fewer Chemical Reactions
}

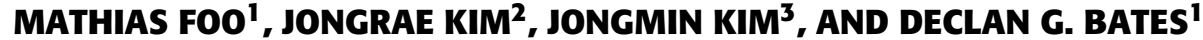 \\ ${ }^{1}$ Warwick Integrative Synthetic Biology Centre, School of Engineering, University of Warwick, Coventry CV4 7AL, U.K. \\ ${ }^{2}$ School of Mechanical Engineering, University of Leeds, Leeds LS2 9JT, U.K. \\ ${ }^{3}$ Wyss Institute for Biologically Inspired Engineering, Harvard University, Boston, MA 02115, USA \\ CORRESPONDING AUTHOR: M. FOO (M.Foo@warwick.ac.uk)
}

This work was supported in part by EPSRC and BBSRC under Grant BB/M017982/1 and in part by the School of Engineering, University of Warwick.

\begin{abstract}
We consider the design of synthetic embedded feedback circuits that can implement desired changes in the concentration of the output of a biomolecular process (reference tracking in control terminology). Such systems require the use of a "subtractor" to generate an error signal that captures the difference between the current and desired values of the process output. Unfortunately, standard implementations of the subtraction operator using chemical reaction networks are one sided, i.e., they cannot produce negative error signals. Previous attempts to deal with this problem by representing signals as the difference in concentrations of two different biomolecular species lead to a doubling of the number of chemical reactions required to generate the circuit, hence sharply increasing the difficulty of experimental implementations and limiting the complexity of potential designs. Here, we propose an alternative approach that introduces a degradation term into the classical proportion-integral (PI) control scheme. The extra tuning flexibility of the PI degradation controller compensates for the limitations of the one-sided subtraction operator, providing robust high-performance tracking of concentration changes with a minimal number of chemical reactions.
\end{abstract}

INDEX TERMS Chemical reaction network, proportional-integral degradation (PI-Deg) controller, synthetic biology.

\section{INTRODUCTION}

A FUNDAMENTAL challenge in the design of synthetic circuitry is to develop designs for feedback systems that can be used to make the outputs of biomolecular processes track desired changes in the concentrations of molecular species of interest [1]. A promising framework for the design of such circuits is provided by nucleic acid-based chemistry, which allows circuits to be designed using abstract chemical reaction theory (see [2] and [3]) and then implemented in deoxyribonucleic acid (DNA) using strand displacement reactions [4]. An essential prerequisite for the tracking of reference signals is the ability to generate an error signal (the difference between the desired and actual values of the process output), which can be used by the controller to drive the process toward its desired state. Although the computation of such error signals is essentially trivial in engineering control systems implemented in silico, this is not the case in standard chemical reaction network theory, which does not yet allow the realization of a proper two-sided biomolecular subtraction operator, i.e., an operator that computes the difference between two inputs regardless of their relative magnitude. This limitation represents a particular problem for feedback control systems, as the error between the desired reference $(r)$ and the output $(y)$ can take both positive $(r>y)$ and negative $(r<y)$ values. Since the controller acts directly on this error signal, the discrepancy in its computation will inevitably result in poor quality tracking and possibly even instability.

To date, the only available solution to this problem is to adopt the design framework proposed in [5], which requires each signal in the circuit to be implemented as the difference in the concentration of two chemical species. As we show below, while this allows the implementation of a two-sided subtractor, it also essentially doubles the number of chemical reactions required to implement the circuit. This represents a major challenge from the point of experimental implementations and places serious constraints on the scala- 
bility of proposed designs. For example, for a circuit whose implementation requires $n$ species, the potential bimolecular crosstalk interactions increase by $n^{2}$. This has prompted researchers to look into ways to reduce crosstalk, for instance, by requiring a certain number of mismatches for any two distinct recognition domains (see [6]). In practice, however, obtaining large numbers of well-behaved sequences with long domains will be extremely challenging.

\section{BIOMOLECULAR SUBTRACTION OPERATORS A. ONE-SIDED SUBTRACTION OPERATOR}

All existing designs for biomolecular subtraction operator using standard chemical reaction network theory implement only a one-sided subtraction operator. For example, Buisman et al. [7] present a comprehensive list and detailed analyses of several mathematical operators, including the subtraction operator, which may be designed using the following abstract chemical reactions:

$$
\begin{aligned}
& x_{i, 1} \stackrel{\gamma}{\rightarrow} x_{i, 1}+x_{o}, \quad x_{\mathrm{itd}}+x_{o} \stackrel{\gamma}{\rightarrow} \emptyset \\
& x_{i, 2} \stackrel{\gamma}{\rightarrow} x_{i, 2}+x_{\mathrm{itd}}, \quad x_{o} \stackrel{\gamma}{\rightarrow} \emptyset .
\end{aligned}
$$

Note that this subtraction operator requires four abstract chemical reactions. Abstract chemical reactions can be represented as ordinary differential equations (ODEs) using generalized mass-action kinetics (see [8]), and the corresponding ODEs for (1) are given by

$$
\begin{aligned}
\frac{d x_{o}}{d t} & =\gamma\left(x_{i, 1}-x_{o} x_{\mathrm{itd}}-x_{o}\right) \\
\frac{d x_{\mathrm{itd}}}{d t} & =\gamma\left(-x_{o} x_{\mathrm{itd}}+x_{i, 2}\right) .
\end{aligned}
$$

At steady state, $x_{i, 2}=x_{o} x_{\mathrm{itd}}$, leading to $x_{o}=x_{i, 1}-x_{i, 2}$. In their analysis of the Jacobian matrix of the ODEs relating to the subtraction operator, Buisman et al. [8] show that when the subtraction of two components results in either a negative value or zero, the system converges to an unstable fixed point or the fixed point does not exist, respectively. Thus, $x_{o}=x_{i, 1}-x_{i, 2}$ when $x_{i, 1} \geq x_{i, 2}$ and $x_{o}=0$ when $x_{i, 1}<x_{i, 2}$, making the subtraction one sided.

In [9] and [10], a subtraction operator was designed using chemical reaction network theory to compute the difference of molecular fluxes, rather than concentrations. This subtraction operator is also one sided, however.

\section{B. TWO-SIDED SUBTRACTION OPERATOR}

An exception to the limitations discussed above is the design framework proposed in [5]. To get around the issue of one-sided subtraction, they represent a signal $u$ as a difference between two chemical species resulting in the chemical species having positive and negative components, i.e., $u:=u^{+}-u^{-}$. This allows the implementation of a two-sided subtraction operator. To illustrate this, consider first the summation operator, whose abstract chemical reactions are given by

$$
\begin{aligned}
& x_{i, 1}^{+} \stackrel{\gamma}{\rightarrow} x_{i, 1}^{+}+x_{o}^{+}, \quad x_{i, 1}^{-} \stackrel{\gamma}{\rightarrow} x_{i, 1}^{-}+x_{o}^{-}, x_{i, 1}^{+}+x_{i, 1}^{-} \stackrel{\eta}{\rightarrow} \emptyset \\
& x_{i, 2}^{+} \stackrel{\gamma}{\rightarrow} x_{i, 2}^{+}+x_{o}^{+}, \quad x_{i, 2}^{-} \stackrel{\gamma}{\rightarrow} x_{i, 2}^{-}+x_{o}^{-}, x_{i, 2}^{+}+x_{i, 2}^{-} \stackrel{\eta}{\rightarrow} \emptyset \\
& x_{o}^{+} \stackrel{\gamma}{\rightarrow} \emptyset, \quad x_{o}^{-} \stackrel{\gamma}{\rightarrow} \emptyset, x_{o}^{+}+x_{o}^{-} \stackrel{\eta}{\rightarrow} \emptyset
\end{aligned}
$$

where $x_{i, 1}$ and $x_{i, 2}$ are the two inputs and $x_{o}$ is the output. $\gamma$ and $\eta$ are reactions rates with $\eta \gg \gamma$. The corresponding ODEs are given by

$$
\begin{aligned}
\frac{d x_{o}^{+}}{d t} & =\gamma\left(x_{i, 1}^{+}+x_{i, 2}^{+}-x_{o}^{+}\right)-\eta x_{o}^{+} x_{o}^{-} \\
\frac{d x_{o}^{-}}{d t} & =\gamma\left(x_{i, 1}^{-}+x_{i, 2}^{-}-x_{o}^{-}\right)-\eta x_{o}^{+} x_{o}^{-} \\
\frac{d x_{o}}{d t} & =\frac{d x_{o}^{+}}{d t}-\frac{d x_{o}^{-}}{d t}=\gamma\left(x_{i, 1}+x_{i, 2}-x_{o}\right)
\end{aligned}
$$

where at steady state (i.e., $d x_{o} / d t=0$ ), $x_{o}=x_{i, 1}+x_{i, 2}$.

Now, for the subtraction operator, its chemical reactions are given by

$$
\begin{aligned}
& x_{i, 1}^{+} \stackrel{\gamma}{\rightarrow} x_{i, 1}^{+}+x_{o}^{+}, \quad x_{i, 1}^{-} \stackrel{\gamma}{\rightarrow} x_{i, 1}^{-}+x_{o}^{-}, x_{i, 1}^{+}+x_{i, 1}^{-} \stackrel{\eta}{\rightarrow} \emptyset \\
& x_{i, 2}^{+} \stackrel{\gamma}{\rightarrow} x_{i, 2}^{+}+x_{o}^{-}, \quad x_{i, 2}^{-} \stackrel{\gamma}{\rightarrow} x_{i, 2}^{-}+x_{o}^{+}, x_{i, 2}^{+}+x_{i, 2}^{-} \stackrel{\eta}{\rightarrow} \emptyset \\
& x_{o}^{+} \stackrel{\gamma}{\rightarrow} \emptyset, \quad x_{o}^{-} \stackrel{\gamma}{\rightarrow} \emptyset, x_{o}^{+}+x_{o}^{-} \stackrel{\eta}{\rightarrow} \emptyset .
\end{aligned}
$$

Note the difference between the superscripts + and - in the abstract chemical reaction compared with (3). The corresponding ODEs are given by

$$
\begin{aligned}
\frac{d x_{o}^{+}}{d t} & =\gamma\left(x_{i, 1}^{+}+x_{i, 2}^{-}-x_{o}^{+}\right)-\eta x_{o}^{+} x_{o}^{-} \\
\frac{d x_{o}^{-}}{d t} & =\gamma\left(x_{i, 1}^{-}+x_{i, 2}^{+}-x_{o}^{-}\right)-\eta x_{o}^{+} x_{o}^{-} \\
\frac{d x_{o}}{d t} & =\frac{d x_{o}^{+}}{d t}-\frac{d x_{o}^{-}}{d t}=\gamma\left(x_{i, 1}-x_{i, 2}-x_{o}\right)
\end{aligned}
$$

where at steady state, $x_{o}=x_{i, 1}-x_{i, 2}$. Both the summation and subtraction operators in (3) and (5) require nine abstract chemical reactions.

Now, note that an operator to sum two concentrations [equivalent to (4) with positive signals] could also have been obtained without using the positive/negative components formalism, by employing the following three chemical reactions: $x_{i, 1} \stackrel{\gamma}{\rightarrow} x_{i, 1}+x_{o}, x_{i, 2} \stackrel{\gamma}{\rightarrow} x_{i, 2}+x_{o}$, and $x_{o} \stackrel{\gamma}{\rightarrow} \emptyset$. Surprisingly, however, it is not possible to obtain an equivalent of the subtraction operator (6) in the same way, as there are no associated abstract chemical reactions to realize it. This can be demonstrated as follows.

Consider the following two reactions: $x_{i, 1} \stackrel{\gamma}{\rightarrow} x_{i, 1}+y$ and $y \stackrel{\gamma}{\rightarrow} \emptyset$. The ODEs for those two reactions are $d y / d t=$ $+\gamma x_{i, 1}$ and $d y / d t=-\gamma y$, respectively, and the final ODE expression can be obtained by summing these two together, i.e., $d y / d t=\gamma\left(x_{i, 1}-y\right)$. Now, the ODE required for the subtraction operator is given by $d y / d t=\gamma\left(x_{i, 1}-x_{i, 2}-y\right)$. We have already shown how we can obtain $d y / d t=\gamma\left(x_{i, 1}-y\right)$, and thus we simply need another abstract chemical reaction that will provide $d y / d t=-\gamma x_{i, 2}$. With the sign on the right-hand side (RHS) of the ODE being negative, one would expect to write $y$ on the left-hand side (LHS) of the abstract chemical reaction. Additionally, we require the multiplication of $x_{i, 2}$ with $\gamma$, which means $x_{i, 2}$ has to be on the LHS of the abstract chemical reaction as well. A natural first attempt would therefore be to write $x_{i, 2}+y \stackrel{\gamma}{\rightarrow} \emptyset$. However, a sum of reactants leads to multiplication in the corresponding ODE, i.e., $d y / d t=$ $-\gamma x_{i, 2} y$. If we are to move $y$ to the RHS of the abstract 
chemical reaction, i.e., $x_{i, 2} \stackrel{\gamma}{\rightarrow} x_{i, 2}+y$, its ODE would be $d y / d t=+\gamma x_{i, 2}$. Thus, there is no way to realize $d y / d t=-\gamma x_{i, 2}$ using standard abstract chemical reactions. This is why it is necessary to use the positive/negative component formalism introduced by [5] in order to realize a two-sided subtraction operator.

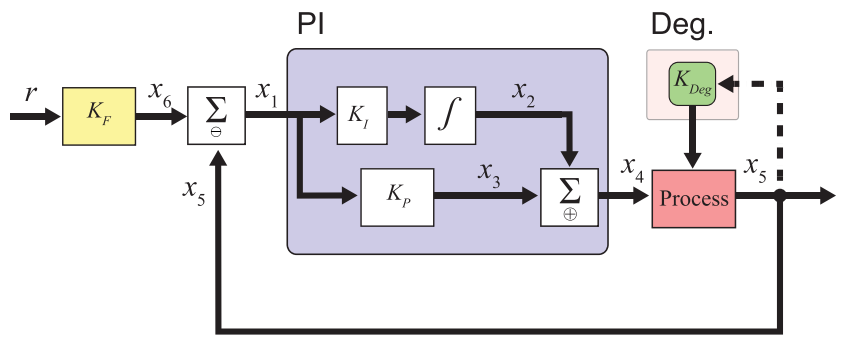

FIGURE 1. System block diagram with the PI-Deg controller. The standard PI controller is shown within the blue rectangular box. The dotted line indicates the additional degradation component with its tuning parameter, $K_{\text {Deg }}$ shown within the pink rectangular box.

\section{PI-Deg CONTROLLER DESIGN WITH ONE-SIDED SUBTRACTION}

The system block diagram for our proposed controller is shown in Fig. 1. A standard PI controller can be described by two scalar gain operators, one integration operator and one summation operator. For the details of the abstract chemical reactions describing those operators, see [5] and [11]. We assume that the feedback control system uses a one-sided subtraction operator. The controller operates on a nonlinear second-order biomolecular process as indicated in Fig. 1. Following the variables defined in Fig. 1, we have the following chemical reactions.

Subtraction: $x_{6} \stackrel{k_{s 1}}{\longrightarrow} x_{6}+x_{1}, x_{\mathrm{itd}}+x_{1} \stackrel{k_{s 1}}{\longrightarrow} \emptyset, x_{5} \stackrel{k_{s 1}}{\longrightarrow} x_{5}+x_{\mathrm{itd}}$, and $x_{1} \stackrel{k_{s 1}}{\longrightarrow} \emptyset$, where $x_{\text {itd }}$ is an intermediate species and $k_{s 1}$ is the subtraction reaction rate.

PI Controller: $x_{1} \stackrel{K_{I}}{\longrightarrow} x_{1}+x_{2}, x_{1} \stackrel{\gamma_{G} K_{P}}{\longrightarrow} x_{1}+x_{3}$ and $x_{3} \stackrel{\gamma_{G}}{\longrightarrow} \emptyset$, $x_{2} \stackrel{k_{s 2}}{\longrightarrow} x_{2}+x_{4}, x_{3} \stackrel{k_{s 2}}{\longrightarrow} x_{3}+x_{4}$, and $x_{4} \stackrel{k_{s 2}}{\longrightarrow} \emptyset$ where $K_{I}$ is the integral gain, $K_{P}$ is the proportional gain, $\gamma_{G}$ is the gain reaction rate, and $k_{s 2}$ is the summation reaction rate.

Process: The chemical reactions implementing the process are given by $x_{4}+x_{e} \stackrel{k_{r 1}}{\longrightarrow} x_{i}, x_{i} \stackrel{k_{r 2}}{\longrightarrow} x_{5}+x_{e}$, and $x_{5} \stackrel{k_{r 3}}{\longrightarrow} \emptyset$, where $k_{r 1}, k_{r 2}$, and $k_{r 3}$ are, respectively, the process binding, catalytic, and degradation rates. $x_{i}$ and $x_{e}$ are intermediate species variables.

To compensate for the limitations of the one-sided subtraction operator, we introduce an additional degradation reaction, with rate $K_{\mathrm{Deg}}$, that acts on the process output as indicated in Fig. 1 by the pink rectangular box. The additional abstract chemical reaction for the degradation term in the process output is given by $x_{5} \stackrel{K_{\text {Deg }}}{\longrightarrow} \emptyset$. We use $K_{\text {Deg }}$ as an additional tuning parameter to the proportional and integral gains, $K_{P}$ and $K_{I}$, of the standard PI controller. The desired change in the concentration of the process output $x_{5}$ specified by the reference signal $r$ is translated into a change in the concentration of species $x_{6}$ via the constant scaling factor $K_{F}$.

The corresponding ODEs for each module of the closed-loop system are then given by the following.
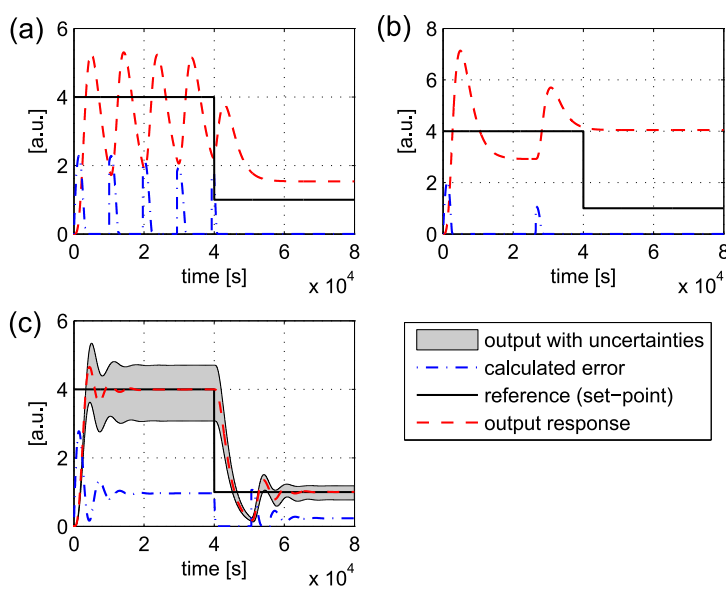

FIGURE 2. System responses with different control gains. (a) Original PI gain: initial PI gain with no prefilter and degradation terms. (b) Tuned PI gain: the PI controller is retuned with no prefilter and degradation terms. (c) PI-Deg: the PI controller incorporating the prefilter and the degradation terms.

\section{Subtraction:}

$$
\sum_{\ominus}:\left\{\begin{array}{l}
\frac{d x_{1}}{d t}=k_{s 1}\left(x_{6}-x_{1} x_{\mathrm{itd}}-x_{1}\right) \\
\frac{d x_{\mathrm{itd}}}{d t}=k_{s 1}\left(x_{5}-x_{1} x_{\mathrm{itd}}\right) .
\end{array}\right.
$$

At steady state, $x_{1}=x_{6}-x_{5}$ for $x_{6} \geq x_{5}$, or $x_{1}=0$ otherwise. PI-Deg controller + Process:

$$
\text { PI: }\left\{\begin{array}{l}
\frac{d x_{2}}{d t}=K_{I} x_{1} \\
\frac{d x_{3}}{d t}=\gamma_{G} K_{P} x_{1}-\gamma_{G} x_{3} \\
\frac{d x_{4}}{d t}=k_{s 2}\left(x_{2}+x_{3}-x_{4}\right) .
\end{array}\right.
$$

At steady state, $x_{4}=x_{2}+x_{3}$, while the second-order biomolecular system considered in this letter is given by

$$
\text { Process: }\left\{\begin{array}{l}
\frac{d x_{i}}{d t}=k_{r 1} x_{4} x_{e}-k_{r 2} x_{i} \\
\frac{d x_{5}}{d t}=k_{r 2} x_{i}-\left(k_{r 3}+K_{\text {Deg }}\right) x_{5}
\end{array}\right.
$$

where $x_{e}+x_{i}=x_{e, T}$ is constant. Here, we are assuming that the natural degradation rate $k_{r 3}$ of the species $x_{5}$ in the process cannot be altered, necessitating the introduction of an additional degradation mechanism represented by $K_{\text {Deg }}$. Note that this design approach can be applied to any process that can be modeled using chemical reaction networks.

\section{SIMULATION RESULTS}

Example simulation results for the standard PI controller with a one-sided subtractor are shown in Fig. 2(a), where $k_{s 1}=3$, $k_{s 2}=4 \times 10^{-4}, \gamma_{G}=8 \times 10^{-4}, k_{r 1}=5 \times 10^{-5}, k_{r 2}=1.6$, $k_{r 3}=8 \times 10^{-4}$, and $x_{e, T}=5.5$, and the reference signal $r$ initially changes from 0 to 4 before decreasing to 1 at time $40000 \mathrm{~s}$. All units are assumed to be defined appropriately. Initial values for $K_{P}$ and $K_{I}$ were chosen as 20 and $2.5 \times 10^{-4}$, respectively. As shown in Fig. 2(a), the output response is highly oscillatory and its convergence rate is very slow. When 
the reference value changes from 4 to $1, r=x_{6}<x_{5}$, the one-sided subtraction operator produces $x_{1}=0$, and the controller is unable to respond to the desired change in the value of $x_{5}$.

To improve the performance of the system, the PI gains were retuned, following standard control theoretic design principles. Example results are shown in Fig. 2(b) for $K_{P}$ increased by a factor of 1.5 and $K_{I}$ increased by a factor of 10. Although the oscillatory behavior has been removed, large overshoots and steady-state errors are now observed. The calculated error by the one-sided subtraction operator converges to zero even for a nonzero error in the output because the subtractor only works correctly when $x_{6} \geq x_{5}$ and outputs zero when $x_{6}<x_{5}$. As a result, the PI controller considers that the output has converged to the desired reference value, when in fact it has not. Repeated attempts to retune the standard PI controller produced no improvement in performance, highlighting the problems caused by the one-sided subtraction operator.

In contrast, achieving effective tracking of reference signals using our PI-Deg controller architecture was quite straightforward. Setting $K_{I}=5 \times 10^{-6}, K_{P}=30$, $K_{F}=1.24$, and $K_{\text {Deg }}=1.2 \times 10^{-3}$ produced the results shown in Fig. 2(c) - the oscillatory behavior is removed, the convergence rate is much faster, and the steady state error is now zero. The use of the degradation term allows us to use a much higher proportional gain and lower integral gain without incurring large overshoots, and now the output of the process is able to track both increasing and decreasing reference changes equally well.

The implementation of the summation and subtraction operations requires identical reaction rates (i.e., $k_{s 1}$ and $k_{s 2}$ ), which may not be feasible in practice. To investigate the effect of this, we performed a robustness analysis as follows. To test the robustness of the PI-Deg controller, we performed Monte Carlo simulations, where we randomly draw all the parameters in the equations implementing the closed-loop system from a uniform distribution and perform repeated simulations. The number of Monte Carlo simulation needed to obtain various levels of estimation uncertainty with known probability are calculated based on the well-known Chernoff bound [12]. A total number of 1060 simulations [12], [13] are required for the Monte Carlo simulation to achieve an accuracy level of 0.05 with a confidence level of $99 \%$. Here, we vary all parameters within ranges of $10 \%$ around their nominal values. Mathematically, we have $p(1+0.1 \delta)$, where $p \in\left\{k_{s 1 i}, \gamma_{G j}, k_{s 2 k}, K_{I}, K_{P}, k_{r 1}, k_{r 2}, k_{r 3}, K_{F}, K_{\text {Deg }}\right\}, \delta$ is a random number from the uniform distribution in $[-1,1]$, $i \in\{a, b, c, d, e\}, j \in\{a, b\}$, and $k \in\{a, b, c\}$. Note that we split reaction rates $k_{s 1}, \gamma_{G}$ and $k_{s 2}$ according to the number of chemical reactions in which they are involved. As shown in Fig. 2(c), the gray shaded region is the output envelope covering all possible response from 1060 Monte Carlo simulations for randomly perturbed parameters in the range of $\pm 10 \%$ from the nominal values. The PI-Deg controller shows a good level of robust performance with no loss of stability due to parameter variations.

The closed-loop feedback control system utilizing our proposed design approach uses a total of 14 abstract chemical reactions compared with a total of 36 if the design framework of [5] using a two-sided subtraction operator is used, a reduction in circuit complexity of $61 \%$. For the DNA strand displacement (DSD) implementation of the proposed PI-Deg controller, the degradation mechanism can be achieved by incorporating auxiliary DNA species specifically designed for the degradation reaction.

\section{CONCLUSION}

Subtraction operators are essential modules in any feedback control system that is designed to track changing reference demands on process outputs, but two-sided subtraction operators have yet to be realized using standard chemical reaction network theory. Current solutions to this problem result in large numbers of additional chemical reactions being required, which complicates experimental implementations and places strong limitations on the scalability of synthetic feedback control systems. We demonstrated a novel feedback controller architecture that compensates for the limitations of a one-sided subtraction operator by introducing an additional degradation term that may be tuned during the controller design process. The resulting PI-Deg controller is shown in simulation to provide accurate and robust tracking of dynamic reference demands, while requiring approximately half as many chemical reactions to implement as current solutions.

\section{REFERENCES}

[1] V. Hsiao, E. L. C. de los Santos, W. R. Whitaker, J. E. Dueber, and R. M. Murray, "Design and implementation of a biomolecular concentration tracker," ACS Synth. Biol., vol. 4, no. 2, pp. 150-161, 2015 .

[2] D. Soloveichik, G. Seelig, and E. Winfree, "DNA as a universal substrate for chemical kinetics," Proc. Nat. Acad. Sci. USA, vol. 107, no. 12, pp. 5393-5398, 2010.

[3] E. D. Sontag and A. Singh, "Exact moment dynamics for feedforward nonlinear chemical reaction networks," IEEE Life Sci. Lett., vol. 1, no. 2 , pp. 26-29, Aug. 2015.

[4] Y.-J. Chen et al., "Programmable chemical controllers made from DNA," Nature Nanotechnol., vol. 8, pp. 755-762, Mar. 2013.

[5] K. Oishi and E. Klavins, "Biomolecular implementation of linear I/O systems," IET Syst. Biol., vol. 5, no. 4, pp. 252-260, Jul. 2011

[6] L. Qian and E. Winfree, "A simple DNA gate motif for synthesizing largescale circuits," J. Roy. Soc. Interface, rsif20100729, 2011.

[7] H. J. Buisman, H. M. M. ten Eikelder, P. A. J. Hilbers, and A. M. L. Liekens, "Computing algebraic functions with biochemical reaction networks," Artif. Life, vol. 15, no. 1, pp. 5-19, 2009

[8] M. Feinberg, "Chemical reaction network structure and the stability of complex isothermal reactors-I. The deficiency zero and deficiency one theorems," Chem. Eng. Sci., vol. 42, pp. 2229-2268, 1986.

[9] M. Bilotta, C. Cosentino, D. G. Bates, and F. Amato, "Retroactivity analysis of a chemical reaction network module for the subtraction of molecular fluxes," in Proc. 37th Annu. Int. Conf. IEEE Eng. Med. Biol. Conf., Milan, Italy, Aug. 2015, pp. 941-944.

[10] C. Cosentino, R. Ambrosino, M. Ariola, M. Bilotta, A. Pironti, and F. Amato, "On the realization of an embedded subtractor module for the control of chemical reaction networks," IEEE Trans. Autom. Control, vol. 61, no. 11, pp. 3638-3643, Nov. 2016.

[11] B. Yordanov, J. Kim, R. L. Petersen, A. Shudy, V. V. Kulkarni, and A. Philips, "Computational design of nucleic acid feedback control circuits," ACS Synth. Biol., vol. 3, no. 8, pp. 600-616, 2014.

[12] M. Vidyasagar, "Statistical learning theory and randomized algorithms for control," IEEE Control Syst., vol. 18, no. 6, pp. 69-85, Dec. 1998.

[13] P. P. Menon, I. Postlethwaite, S. Bennani, A. Marcos, and D. G. Bates, "Robustness analysis of a reusable launch vehicle flight control law,' Control Eng. Pract., vol. 17, no. 7, pp. 751-765, 2009. 